\title{
Enhanced Surrogate Models for Statistical Design Exploiting Space Mapping Technology
}

\author{
Slawomir Koziel, Member, IEEE, John W. Bandler, Fellow, IEEE, \\ Ahmed S. Mohamed, Student Member, IEEE, and Kaj Madsen \\ McMaster University, Hamilton, ON, L8S4K1, Canada www.sos.mcmaster.ca
}

\begin{abstract}
We present advances in microwave and RF device modeling exploiting Space Mapping (SM) technology. We propose new SM modeling formulations utilizing input mappings, output mappings, frequency scaling and quadratic approximations. Our aim is to enhance circuit models for statistical analysis and yield-driven design. We illustrate our results using a capacitively-loaded two-section impedance transformer, a single-resonator waveguide filter and a six-section H-plane waveguide filter.
\end{abstract}

Index Terms-CAD, EM modeling, modeling techniques, microwave filters, space mapping, surrogate modeling.

\section{INTRODUCTION}

Full-wave EM simulations of microwave structures are CPU intensive. Statistical analysis and yield optimization, crucial for manufacturability-driven designs in a time-tomarket development environment, demand accurate and fast models. The Space Mapping (SM) concept [1]-[2] can help address this issue.

SM assumes the existence of "fine" and "coarse" models. The "fine" model may be a high fidelity CPU-intensive EM simulator which may not be desirable for direct statistical analysis and design. The "coarse" model can be a simplified representation such as an equivalent circuit with empirical formulas. SM modeling techniques [3], [4], [5] exploit the speed of the coarse model and the accuracy of the fine model to develop fast, accurate enhanced models valid over a wide range of parameter values.

Our new SM modeling formulations utilize input mappings, output mappings, frequency scaling and quadratic approximations. Examples include a single-resonator waveguide filter and a six-section H-plane waveguide filter. The filters exploit MEFiSTo [6] as the fine (validation) model using MEFiSTo's electromagnetically oriented rubber cell feature to perform response interpolation.

This work was supported in part by the Natural Sciences and Engineering Research Council of Canada under Grants OGP0007239 and STGP269760, and by Bandler Corporation. A.S. Mohamed is supported by an Ontario Graduate Scholarship (OGS).

S. Koziel, J.W. Bandler and A.S. Mohamed are with the Simulation Optimization Systems Research Laboratory, Department of Electrical and Computer Engineering, McMaster University, Hamilton, ON, Canada L8S 4K1

J.W. Bandler is also with Bandler Corporation, Dundas, ON, Canada L9H 5E7.

K. Madsen is with Informatics and Mathematical Modelling, Technical University of Denmark, DK-2800, Lyngby, Denmark.

\section{THE MODELS}

Let $\boldsymbol{R}_{f}: X_{f} \rightarrow R^{m}$ and $\boldsymbol{R}_{c}: X_{c} \rightarrow R^{m}$ denote the fine and coarse model response vectors, where $X_{f} \subseteq R^{n}$ and $X_{c} \subseteq R^{n}$ are design variable domains of the fine and coarse models, respectively. For example, $\boldsymbol{R}_{f}(\boldsymbol{x})$ and $\boldsymbol{R}_{c}(\boldsymbol{x})$ may represent the magnitude of a transfer function at $m$ chosen frequencies.

We denote by $X_{R} \subseteq X_{f}$ the region of interest in which we want enhanced matching between the surrogate and the fine model. We assume that $X_{R}$ is an $n$-dimensional interval in $R^{n}$ with center at reference point $\boldsymbol{x}^{0}=\left[\begin{array}{lll}x_{0.1} & \ldots & x_{0 . n}\end{array}\right]^{T} \in R^{n}$ :

$$
X_{R}=\left[\boldsymbol{x}^{0}-\boldsymbol{\delta}, \boldsymbol{x}^{0}+\boldsymbol{\delta}\right]=\left[x_{0.1}-\delta_{1}, x_{0.1}+\delta_{1}\right] \times \ldots \times\left[x_{0, n}-\delta_{n}, x_{0, n}+\delta_{n}\right]
$$

where $\boldsymbol{\delta}=\left[\delta_{1} \ldots \delta_{n}\right]^{T} \in R_{+}^{n}$ determines the size of $X_{R}$. We use $X_{R}\left(x^{0}, \delta\right)$ to denote the region of interest defined by $\boldsymbol{x}^{0}$ and $\delta$.

The number of fine model evaluations used to construct a surrogate should be small since we assume that each evaluation is expensive. But, we have to account for dimension of the design variable space. We use the set of evaluation points (also called the base set) denoted by $X_{B}$, to consist of $2 n+1$ points, where $n=\operatorname{dim}\left(X_{f}\right)$. We have

$$
X_{B}=\left\{\boldsymbol{x}^{0}, \boldsymbol{x}^{1}, \ldots, \boldsymbol{x}^{2 n}\right\}
$$

where $\boldsymbol{x}^{0}$ is the reference point, and

$$
\boldsymbol{x}^{j}=\boldsymbol{x}^{0}+(-1)^{j} \delta_{\lceil j / 2\rceil} \cdot \boldsymbol{u}_{\lceil j / 2\rceil}, \quad j=1, \ldots, 2 n
$$

where $\boldsymbol{u}_{k}=\left[\begin{array}{lllllll}0 & \ldots & 0 & 1 & 0 & \ldots & 0\end{array}\right]^{T}$ is a unit vector with 1 at $k$ th position; $\delta_{k}$ is the size of the region $X_{R}$ along the $k$ th axis. This distribution of base points is called the star distribution [4].

Our basic SM-based surrogate model $\boldsymbol{R}_{s 1}: X_{f} \rightarrow R^{m}$ is defined as

$$
\boldsymbol{R}_{s 1}(\boldsymbol{x})=\boldsymbol{R}_{c}(\overline{\boldsymbol{B}} \boldsymbol{x}+\overline{\boldsymbol{c}})
$$

where matrices $\overline{\boldsymbol{B}} \in M_{n \times n}$ and $\overline{\boldsymbol{c}} \in M_{n \times 1}$ are obtained using the parameter extraction

$$
(\overline{\boldsymbol{B}}, \overline{\boldsymbol{c}})=\arg \min _{(\boldsymbol{B}, \boldsymbol{c})} \sum_{j=0}^{2 n}\left\|\boldsymbol{R}_{f}\left(\boldsymbol{x}^{j}\right)-\boldsymbol{R}_{c}\left(\boldsymbol{B} \boldsymbol{x}^{j}+\boldsymbol{c}\right)\right\|
$$

$\boldsymbol{R}_{s 1}$ is based on the original SM concept [1].

The second model $\boldsymbol{R}_{s 2}: X_{f} \rightarrow R^{m}$ is defined as

$$
\boldsymbol{R}_{s 2}(\boldsymbol{x})=\overline{\boldsymbol{A}} \cdot \boldsymbol{R}_{c}(\overline{\boldsymbol{B}} \boldsymbol{x}+\overline{\boldsymbol{c}})
$$

where matrices $\overline{\boldsymbol{A}}=\operatorname{diag}\left\{a_{1}, \ldots, a_{m}\right\}, \overline{\boldsymbol{B}} \in M_{n \times n}$ and $\overline{\boldsymbol{c}} \in M_{n \times 1}$ are obtained using the parameter extraction

$$
(\overline{\boldsymbol{A}}, \overline{\boldsymbol{B}}, \overline{\boldsymbol{c}})=\arg \min _{(\boldsymbol{A}, \boldsymbol{B}, \boldsymbol{c})} \sum_{j=0}^{2 n}\left\|\boldsymbol{R}_{f}\left(\boldsymbol{x}^{j}\right)-\boldsymbol{A} \cdot \boldsymbol{R}_{c}\left(\boldsymbol{B} \boldsymbol{x}^{j}+\boldsymbol{c}\right)\right\|
$$

$\boldsymbol{R}_{s 2}$ augments the output SM concept [7] to $\boldsymbol{R}_{s 1}$.

$\boldsymbol{R}_{s 3}: X_{f} \rightarrow R^{m}$ is a variation of $\boldsymbol{R}_{s 2}$ and is defined as

$$
\boldsymbol{R}_{s 3}(\boldsymbol{x})=\overline{\boldsymbol{A}} \cdot \boldsymbol{R}_{c}(\bar{B} \boldsymbol{x}+\overline{\boldsymbol{c}})+\overline{\boldsymbol{q}}(\boldsymbol{x})
$$


with matrices $\overline{\boldsymbol{A}}, \overline{\boldsymbol{B}}$, and $\overline{\boldsymbol{c}}$ obtained by (7). $\boldsymbol{q}(\boldsymbol{x})=\left[\begin{array}{lll}q_{1}(\boldsymbol{x}) & \ldots & q_{m}(\boldsymbol{x})\end{array}\right]^{T}$, is a function that approximates the residuals between the fine model responses and the $\boldsymbol{R}_{s 2}$ model responses using the maximally flat quadratic approximation [8] set up using the residuals at all base points $\boldsymbol{x}^{0}, \boldsymbol{x}^{1}, \ldots, \boldsymbol{x}^{2 n}$.

Model $\boldsymbol{R}_{s 4}$, is an enhancement of $\boldsymbol{R}_{s 2}$, which is defined as in (6), but the coarse model is evaluated at a different frequency than the fine model by using the transformation: $\omega \rightarrow w_{0}+w_{1} \omega$, where $\overline{\boldsymbol{w}}=\left(w_{0}, w_{1}\right) \in R^{2}$ is obtained together with matrices $\overline{\boldsymbol{A}}, \overline{\boldsymbol{B}}$ and $\overline{\boldsymbol{c}}$ using a parameter extraction process similar to (7).

The last model we consider (or, rather, a family of models) is $\boldsymbol{R}_{s 5 . l}$ which consists of $l$ separate $\boldsymbol{R}_{s 2}$ models defined on disjoint frequency sub-bands and considered as a one model. We assume a uniform division of the frequency band. In particular, if $\boldsymbol{R}_{s 2 . i}$ is the $\boldsymbol{R}_{s 2}$ type model defined for the partial fine model response $\boldsymbol{R}_{f, i}(\boldsymbol{x})=\left[R_{f\lfloor\lfloor(i-1) m / l]+1}(\boldsymbol{x}) \ldots R_{f:\lfloor i m / l\rfloor}(\boldsymbol{x})\right]^{T}$, $i=1, \ldots, l$, then we have $\boldsymbol{R}_{s 5 . l}(\boldsymbol{x})=\left[\boldsymbol{R}_{s 2.1}(\boldsymbol{x})^{T} \ldots \boldsymbol{R}_{s 2 . l}(\boldsymbol{x})^{T}\right]^{T}$.

\section{EXAMPLES}

The models are constructed using $2 n+1$ base points ( $n$ is the number of design variables) as described in Section II. For each example, we perform statistical analysis of the model error using the set of test points randomly chosen in the region of interest (with uniform distribution). The error is measured as an $l_{2}$ norm of the difference between the fine and the surrogate model responses.

\section{A. Single Resonator Filter [9]}

Fig. 1 shows a single-resonator filter. Design parameters are resonator length $d$ and septum width $W$. The rectangular waveguide width and length are fixed. The frequency range is $3.0 \mathrm{GHz} \leq \omega \leq 5.0 \mathrm{GHz}$ with a step of $0.04 \mathrm{GHz}$. The reference point is $\boldsymbol{x}^{0}=\left[\begin{array}{lll}33.00 & 14.59\end{array}\right]^{T} \mathrm{~mm}$. The fine model employs MEFiSTo [6] in a 2D mode with $1 \mathrm{~mm} \times 1 \mathrm{~mm}$ mesh size utilizing the rubber cell feature to perform interpolation. The coarse Matlab model consists of lumped inductances and dispersive transmission line sections connected by circuit theory. The inductive susceptances, simplified formulas due to Marcuvitz, are used to model the resonator septa.

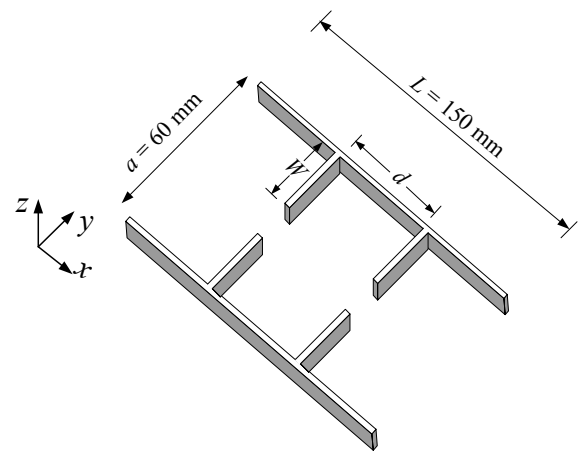

Fig. 1. Topology of the single-resonator filter [9].
We consider two different sizes of the region of interest: $3 \%$ and $10 \%$ deviation from $x^{0}$ (i.e., $\delta=0.03 x^{0}$, and $\delta=0.1 x^{0}$ ). Table I shows the statistical analysis of the modeling error for 100 random test points. Figs. 2 and 3 show error plots for the coarse model $\boldsymbol{R}_{c}$ and model $\boldsymbol{R}_{s 2}$, respectively, for a $10 \%$ region, while Figs. $4 \mathrm{a}$ and $4 \mathrm{~b}$ show $\left|S_{21}\right|$ versus frequency for the fine model ( $\left(\right.$ ), the coarse model ( $\times$ ), and the $\boldsymbol{R}_{s 2}$ model (o) at $\boldsymbol{x}^{0}$ (reference point) and at a test point.

TABLE I

ERROR STATISTICS FOR THE SINGLE RESONATOR FILTER USING 100 RANDOM TEST POINTS IN THE REGION OF INTEREST

\begin{tabular}{ccccc}
\hline \multirow{2}{*}{ Model } & \multicolumn{2}{c}{ Region of interest size 3\% } & \multicolumn{2}{c}{ Region of interest size 10\% } \\
\cline { 2 - 5 } & Mean Error & Max. Error & Mean Error & Max. Error \\
\hline $\boldsymbol{R}_{c}$ & 0.587 & 0.789 & 0.567 & 0.889 \\
$\boldsymbol{R}_{s 1}$ & 0.131 & 0.253 & 0.134 & 0.280 \\
$\boldsymbol{R}_{s 2}$ & 0.118 & 0.247 & 0.128 & 0.280 \\
$\boldsymbol{R}_{s 3}$ & 0.106 & 0.334 & 0.149 & 0.344 \\
$\boldsymbol{R}_{s 4}$ & 0.119 & 0.254 & 0.133 & 0.270 \\
$\boldsymbol{R}_{s 5.2}$ & 0.117 & 0.246 & 0.127 & 0.281 \\
$\boldsymbol{R}_{s 5.3}$ & 0.117 & 0.248 & 0.141 & 0.291 \\
\hline
\end{tabular}

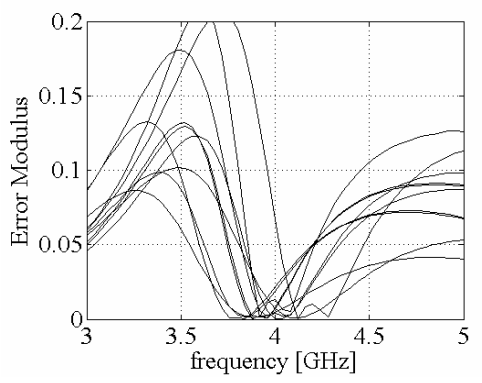

Fig. 2. Error plots for the $\boldsymbol{R}_{c}$ model (modulus of the difference between $\boldsymbol{R}_{f}$ and $\boldsymbol{R}_{c}$ ) for the single resonator filter (10 test points); $10 \%$ region.

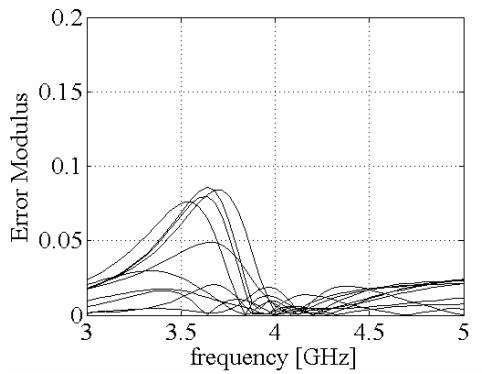

Fig. 3. Error plots for the $\boldsymbol{R}_{s 2}$ model (modulus of the difference between $\boldsymbol{R}_{f}$ and $\boldsymbol{R}_{s 2}$ ) for the single resonator filter (10 test points); $10 \%$ region.

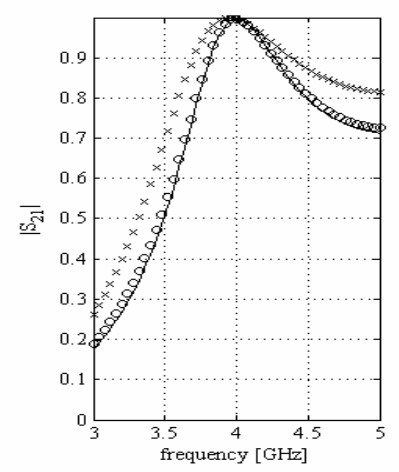

(a)

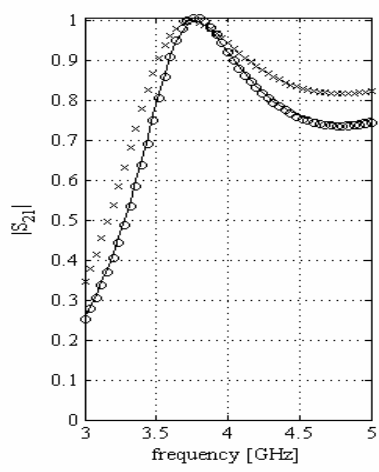

(b)
Fig. 4. $\left|S_{21}\right|$ vs. frequency for the single resonator filter for a $10 \%$ region of interest: the fine model (-), the coarse model ( $\times$ ), and the $\boldsymbol{R}_{s 2}$ model (o); (a) at $\boldsymbol{x}^{0}$, and (b) at a test point. 


\section{B. Capacitively-loaded 10:1 Impedance Transformer [10]}

We consider an ideal two-section transmission line (TL) "coarse" model, and a capacitively-loaded TL "fine" model with capacitors $C_{1}=C_{2}=C_{3}=10 \mathrm{pF}$. See Fig. 5. The electrical lengths $L_{1}$ and $L_{2}$ at $1 \mathrm{GHz}$ are chosen as design parameters. The frequency range is $0.5 \mathrm{GHz} \leq \omega \leq 1.5 \mathrm{GHz}$ with a step of

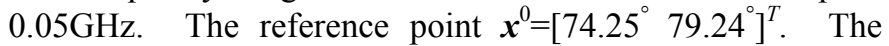
characteristic impedances are kept fixed at the optimal values $Z_{1}=4.4721 \Omega$ and $Z_{2}=2.2361 \Omega$. The fine and coarse models are implemented in Matlab.

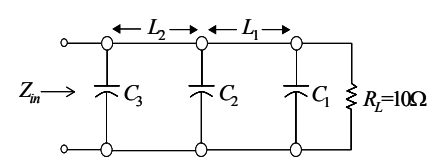

(a)

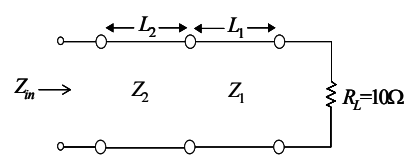

(b)

Fig. 5. Two-section capacitively-loaded 10:1 impedance transformer: (a) fine model, (b) coarse model [10].

As before we consider two sizes of the region of interest, namely, $3 \%$ and $10 \%$ deviation from $\boldsymbol{x}^{0}$. Table II shows the statistical analysis of the modeling error for 100 random test points. Figs. 6-8 show error plots for the coarse model $\boldsymbol{R}_{c}$, the model $\boldsymbol{R}_{s 1}$ and the model $\boldsymbol{R}_{s 2}$, respectively, for a $10 \%$ region.

TABLE II

ERROR STATISTICS FOR THE TWO-SECTION IMPEDANCE TRANSFORMER USING 100 RANDOM TEST POINTS IN THE REGION OF INTEREST

\begin{tabular}{ccccc}
\hline \multirow{2}{*}{ Model } & \multicolumn{2}{c}{ Region of interest size 3\% } & \multicolumn{2}{c}{ Region of interest size 10\% } \\
\cline { 2 - 5 } & Mean Error & Max. Error & Mean Error & Max. Error \\
\hline $\boldsymbol{R}_{c}$ & 0.726 & 0.787 & 0.757 & 0.938 \\
$\boldsymbol{R}_{s 1}$ & 0.072 & 0.082 & 0.090 & 0.146 \\
$\boldsymbol{R}_{s 2}$ & 0.015 & 0.028 & 0.042 & 0.099 \\
$\boldsymbol{R}_{s 3}$ & 0.0025 & 0.011 & 0.021 & 0.061 \\
$\boldsymbol{R}_{s 4}$ & 0.016 & 0.029 & 0.042 & 0.078 \\
$\boldsymbol{R}_{s 5.2}$ & 0.0076 & 0.016 & 0.029 & 0.046 \\
$\boldsymbol{R}_{s 5.3}$ & 0.0062 & 0.015 & 0.025 & 0.048 \\
\hline
\end{tabular}

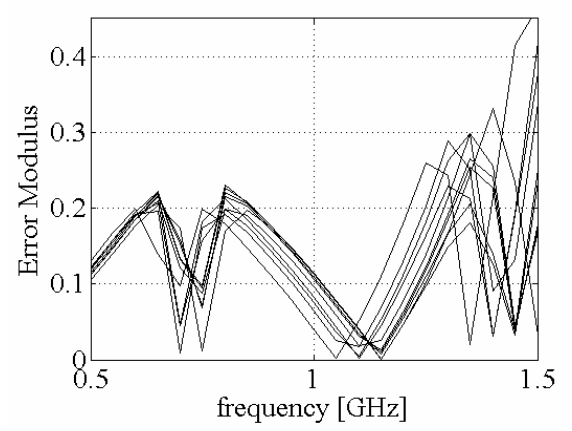

Fig. 6. Error plots for the $\boldsymbol{R}_{c}$ model (modulus of the difference between $\boldsymbol{R}_{f}$ and $\boldsymbol{R}_{\boldsymbol{c}}$ ) for the two-section impedance transformer (10 test points); $10 \%$ region.

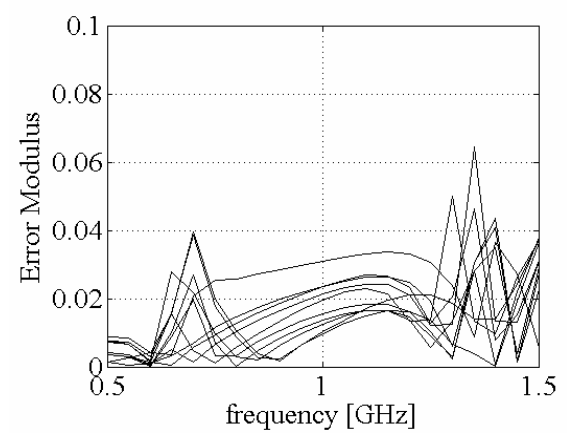

Fig. 7. Error plots for the $\boldsymbol{R}_{s 1}$ model (modulus of the difference between $\boldsymbol{R}_{f}$ and $\boldsymbol{R}_{s 1}$ ) for the two-section impedance transformer (10 test points); $10 \%$ region.

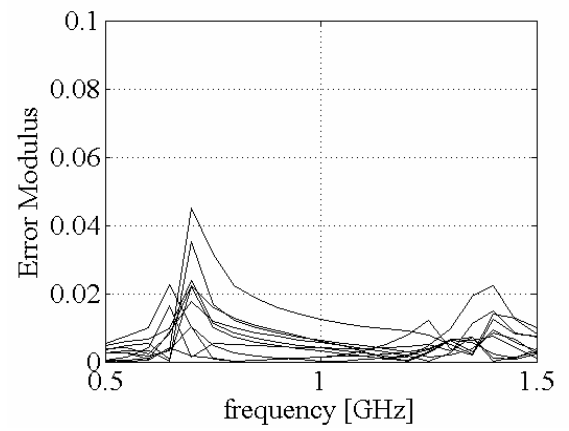

Fig. 8. Error plots for the $\boldsymbol{R}_{s 2}$ model (modulus of the difference between $\boldsymbol{R}_{f}$ and $\boldsymbol{R}_{s 2}$ ) for the two-section impedance transformer (10 test points); $10 \%$ region.

C. Six-Section H-Plane Waveguide Filter [11], [12]

We use a waveguide of width 1.372 inches $(34.85 \mathrm{~mm})$. The six waveguide sections are separated by seven H-plane septa, which have a finite thickness of 0.0245 inches $(0.6223$ $\mathrm{mm})$. Design parameters are the waveguide section lengths $L_{1}, L_{2}$ and $L_{3}$ and the septa widths $W_{1}, W_{2}, W_{3}$ and $W_{4}$ (Fig. 9);

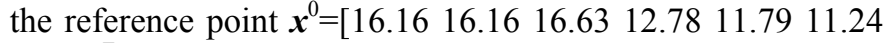
$11.16]^{T} \mathrm{~mm}$. We use 51 points from $5.0 \mathrm{GHz} \leq \omega \leq 10.0 \mathrm{GHz}$.

The fine model is simulated using MEFiSTo [6] in a $2 \mathrm{D}$ mode with $0.6223 \mathrm{~mm} \times 0.6223 \mathrm{~mm}$ mesh size utilizing the rubber cell feature to perform interpolation for the off-grid points. The Matlab coarse model has lumped inductances and dispersive transmission line sections. We simplify formulas due to Marcuvitz for the inductive susceptances corresponding to the H-plane septa. They are connected to the transmission line sections by circuit theory.

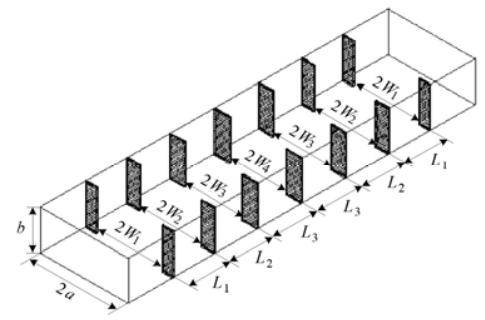

Fig. 9. The six-section H-plane waveguide filter [11], [12].

As before we consider two sizes of the region of interest, namely, $3 \%$ and $10 \%$ deviation from $\boldsymbol{x}^{0}$. Table III shows the statistical analysis of the modeling error for 100 random test 
points. Figs. 10 and 11 show error plots for the models $\boldsymbol{R}_{c}$ and $\boldsymbol{R}_{s 2}$, respectively, for a $10 \%$ region.

TABLE III

ERRor STATISTICS FOR THE SIX-SECTION H-PLANE WAVEGUIDE FILTER USING 100 RANDOM TEST POINTS IN THE REGION OF INTEREST

\begin{tabular}{ccccc}
\hline \multirow{2}{*}{ Model } & \multicolumn{2}{c}{ Region of interest size 3\% } & \multicolumn{2}{c}{ Region of interest size 10\% } \\
\cline { 2 - 5 } & Mean Error & Max. Error & Mean Error & Max. Error \\
\hline $\boldsymbol{R}_{c}$ & 0.636 & 0.739 & 0.695 & 1.123 \\
$\boldsymbol{R}_{s 1}$ & 0.287 & 0.395 & 0.276 & 0.532 \\
$\boldsymbol{R}_{s 2}$ & 0.258 & 0.376 & 0.239 & 0.538 \\
$\boldsymbol{R}_{s 3}$ & 0.257 & 0.454 & 0.244 & 0.556 \\
$\boldsymbol{R}_{s 4}$ & 0.259 & 0.384 & 0.240 & 0.552 \\
$\boldsymbol{R}_{s 5.2}$ & 0.258 & 0.378 & 0.247 & 0.504 \\
$\boldsymbol{R}_{s 5.3}$ & 0.259 & 0.392 & 0.242 & 0.543 \\
\hline
\end{tabular}

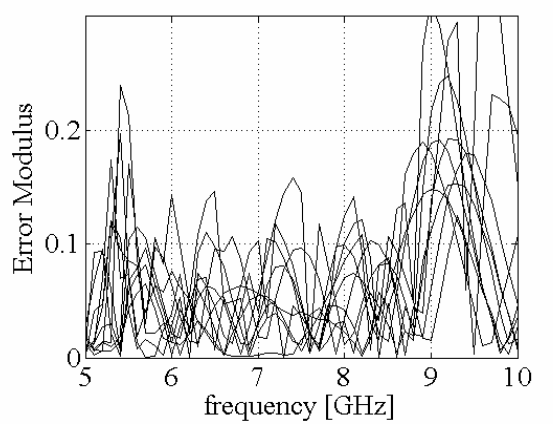

Fig. 10. Error plots for the $\boldsymbol{R}_{c}$ model (modulus of the difference between $\boldsymbol{R}_{f}$ and $\boldsymbol{R}_{\boldsymbol{c}}$ ) for the six-section H-plane waveguide filter (10 test points); $10 \%$ region.

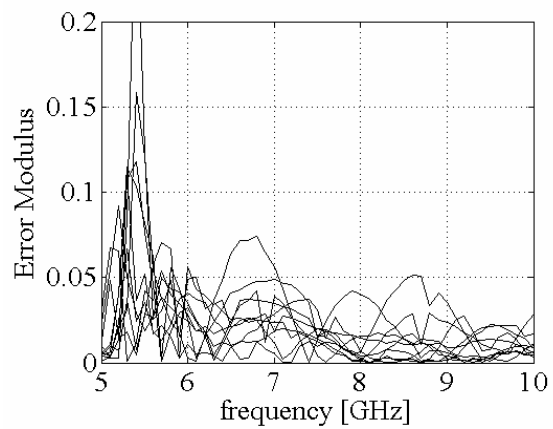

Fig. 11. Error plots for the $\boldsymbol{R}_{s 2}$ model (modulus of the difference between $\boldsymbol{R}_{f}$ and $\boldsymbol{R}_{s 2}$ ) for the six-section H-plane waveguide filter (10 test points); $10 \%$ region.

\section{CONCLUSIONS}

A family of SM-based surrogate models for statistical analysis and yield-driven design is presented and verified. Our experiments show that our enhanced surrogate models using output SM combined with other techniques perform better than the basic SM model employing the original SM by itself.

Besides the already known fact that SM-based surrogates provide good accuracy even for a small amount of fine model data, our results show that the new surrogates $\boldsymbol{R}_{s 2}, \ldots, \boldsymbol{R}_{s 5}$ perform better than $\boldsymbol{R}_{s 1}$ (and, of course, better than the given coarse model $\boldsymbol{R}_{c}$ ). In some cases, quadratic approximations and multiple modeling on frequency sub-bands give further improvement. Lack of improvement in other cases suggests that to improve accuracy we should employ additional fine model information or simply improve the coarse model.

In future, we will develop SM-based surrogate models by combining well-selected and properly utilized additional fine model data with more advanced approximation/interpolation techniques. This should lead to better surrogate models, i.e., ones that allow us to further decrease the modeling error.

\section{ACKNOWLEDGEMENT}

The authors wish to acknowledge Dr. W.J.R. Hoefer, Faustus Scientific Corporation, Victoria, BC, for making the MEFiSTo system available and for assisting in its use. The authors also acknowledge useful technical discussions with Dr. M.H. Bakr, McMaster University, Hamilton, ON, Canada.

\section{REFERENCES}

[1] J.W. Bandler, R.M. Biernacki, S.H. Chen, P.A. Grobelny and R.H. Hemmers, "Space mapping technique for electromagnetic optimization," IEEE Trans. Microwave Theory and Tech., vol. 42, pp. 2536-2544, Dec. 1994.

[2] J.W. Bandler, R.M. Biernacki, S.H. Chen, R.H. Hemmers and K. Madsen, "Electromagnetic optimization exploiting aggressive space mapping," IEEE Trans. Microwave Theory and Tech., vol. 43, pp. 2874-2882, Dec. 1995.

[3] M.H. Bakr, J.W. Bandler and N. Georgieva, "Modeling of microwave circuits exploiting space derivative mapping," in 1999 IEEE MTT-S Int. Microwave Symp. Dig., Anaheim, CA, June 1999, pp. 715-718.

[4] J.W. Bandler, N. Georgieva, M.A. Ismail, J.E. Rayas-Sánchez and Q. J. Zhang, "A generalized space mapping tableau approach to device modeling," IEEE Trans. Microwave Theory and Tech., vol. 49, pp. 67-79, Jan. 2001.

[5] J.W. Bandler, M.A. Ismail, J.E. Rayas-Sánchez and Q.J. Zhang, "Neuromodeling of microwave circuits exploiting space mapping technology," IEEE Trans. Microwave Theory and Tech., vol. 47, pp. 2417-2427, Dec. 1999.

[6] MEFiSTo-3D Pro, version 4.0, Faustus Scientific Corporation, 1256 Beach Drive, Victoria, BC, V8S 2N3, Canada, 2004.

[7] J.W. Bandler, D.M. Hailu, K. Madsen and F. Pedersen, "A space-mapping interpolating surrogate algorithm for highly optimized EM-based design of microwave devices," IEEE Trans. Microwave Theory and Tech., vol. 52, pp. 2593-2600, Nov. 2004.

[8] R.M. Biernacki, J.W. Bandler, J. Song and Q.J. Zhang, "Efficient quadratic approximation for statistical design," IEEE Trans. Circuits and Systems, vol.36, pp.1449-1454, Nov. 1989.

[9] M.H. Bakr, P.P.M. So and W.J.R. Hoefer, "The generation of optimal microwave topologies using time-domain field synthesis," IEEE Trans. Microwave Theory and Tech., vol. 50, pp. 2537-2544, Nov. 2002.

[10] M.H. Bakr, J.W. Bandler, K. Madsen, J.E. Rayas-Sánchez and J. Søndergaard, "Space mapping optimization of microwave circuits exploiting surrogate models," IEEE Trans. Microwave Theory and Tech., vol. 48, pp. 2297-2306, Dec. 2000.

[11] G.L. Matthaei, L. Young and E.M.T. Jones, Microwave filters, Impedance-matching Networks, and Coupling Structures, 1st ed. New York: McGraw-Hill, 1964.

[12] M.H. Bakr, J.W. Bandler, N. Georgieva and K. Madsen, "A hybrid aggressive space mapping algorithm for EM optimization," IEEE Trans. Microwave Theory and Tech., vol. 47, pp. 2440-2449, Dec. 1999. 